\title{
Localized Method of Fundamental Solutions for Three-Dimensional Elasticity Problems: Theory
}

\author{
Yan $\mathrm{Gu}^{1,2, *}$, Chia-Ming $\mathrm{Fan}^{3}$ and Zhuojia Fu ${ }^{4}$ \\ ${ }^{1}$ School of Mathematics and Statistics, Qingdao University, Qingdao, Shandong 266071, \\ China \\ ${ }^{2}$ Department of Civil Engineering, University of Siegen, Paul-Bonatz-Str. 9-11, \\ D-57076 Siegen, Germany \\ ${ }^{3}$ Department of Harbor and River Engineering $\mathcal{E}$ Computation and Simulation Center, \\ National Taiwan Ocean University, Keelung 20224, Taiwan \\ ${ }^{4}$ Center for Numerical Simulation Software in Engineering and Sciences, College of \\ Mechanics and Materials, Hohai University, Nanjing, Jiangsu 211100, China
}

Received 15 May 2020; Accepted (in revised version) 8 December 2020

\begin{abstract}
A localized version of the method of fundamental solution (LMFS) is devised in this paper for the numerical solutions of three-dimensional (3D) elasticity problems. The present method combines the advantages of high computational efficiency of localized discretization schemes and the pseudo-spectral convergence rate of the classical MFS formulation. Such a combination will be an important improvement to the classical MFS for complicated and large-scale engineering simulations. Numerical examples with up to 100,000 unknowns can be solved without any difficulty on a personal computer using the developed methodologies. The advantages, disadvantages and potential applications of the proposed method, as compared with the classical MFS and boundary element method (BEM), are discussed.
\end{abstract}

AMS subject classifications: 62P30, 65M32, 65K05

Key words: Method of fundamental solutions, meshless method, large-scale simulations, elasticity problems.

\section{Introduction}

The method of fundamental solutions (MFS) has emerged as a robust boundary-type meshless method for the solutions of certain boundary value problems [1-8]. The method won the favor of many researchers in engineering and science due to its advantage of high accuracy for many engineering applications [7,9-13]. The classical MFS approach,

*Corresponding author.

Email: guyan1913@163.com (Y. Gu) 
however, produces dense and non-symmetric matrix of algebraic equations that requires memory and other operators to compute the unknown coefficients [14-18]. This makes the method limited to solving small-scale problems with thousands of degrees of freedom for a long time $[7,19-24]$.

To overcome the aforementioned bottleneck associated with the classical MFS, a localized version of the method, named as the localized MFS (LMFS), is proposed by Fan and his coworkers [25-27]. In the LMFS approach, the whole computational domain is divided into a set of overlapping local subdomains in which the classical MFS approximation and moving least square (MLS) techniques are employed. Since the final system of algebraic equations is sparse, the computational efficiency of the method has been fully improved and the method can now be easily used to simulate large-scale applied mechanics problems. This paper documents the first attempt to apply the method for the numerical solutions of 3D elasticity problems. Some possible improvement as well as the influence of several factors on the overall accuracy of the method are also discussed. Numerical examples with up to 100,000 unknowns are solved successfully on a Core (TM) i7 PC using the developed LMFS code. A self-contained Matlab code is provided in the end of the paper.

\section{Statement of the basic problem}

The well-known Cauchy-Navier equations for 3D elasticity problems are [28-30]:

$$
\begin{aligned}
& \left(\frac{2-2 v}{1-2 v}\right) \frac{\partial^{2} u_{1}}{\partial x_{1}^{2}}+\frac{\partial^{2} u_{1}}{\partial x_{2}^{2}}+\frac{\partial^{2} u_{1}}{\partial x_{3}^{2}}+\left(\frac{1}{1-2 v}\right) \frac{\partial^{2} u_{2}}{\partial x_{1} \partial x_{2}}+\left(\frac{1}{1-2 v}\right) \frac{\partial^{2} u_{3}}{\partial x_{1} \partial x_{3}}=0, \\
& \left(\frac{1}{1-2 v}\right) \frac{\partial^{2} u_{1}}{\partial x_{1} \partial x_{2}}+\frac{\partial^{2} u_{2}}{\partial x_{1}^{2}}+\left(\frac{2-2 v}{1-2 v}\right) \frac{\partial^{2} u_{2}}{\partial x_{2}^{2}}+\frac{\partial^{2} u_{2}}{\partial x_{3}^{2}}+\left(\frac{1}{1-2 v}\right) \frac{\partial^{2} u_{3}}{\partial x_{2} \partial x_{3}}=0, \\
& \left(\frac{1}{1-2 v}\right) \frac{\partial^{2} u_{1}}{\partial x_{1} \partial x_{3}}+\left(\frac{1}{1-2 v}\right) \frac{\partial^{2} u_{2}}{\partial x_{2} \partial x_{3}}+\frac{\partial^{2} u_{3}}{\partial x_{1}^{2}}+\frac{\partial^{2} u_{3}}{\partial x_{2}^{2}}+\left(\frac{2-2 v}{1-2 v}\right) \frac{\partial^{2} u_{3}}{\partial x_{3}^{2}}=0,
\end{aligned}
$$

with the following displacement and/or traction boundary conditions:

$$
\begin{array}{ll}
u_{i}=\bar{u}_{i} & \text { on boundary } \Gamma_{u}, \\
t_{i}=\bar{t}_{i} & \text { on boundary } \Gamma_{t},
\end{array}
$$

where $u_{i}$ and $t_{i}$ denote displacements and boundary tractions, respectively, the barred quantities $\bar{u}_{i}$ and $\bar{t}_{i}$ represent known boundary conditions, and $v$ stands for the Poisson's ratio. According to theory of linear elasticity, the strains $\left(\varepsilon_{i j}\right)$ and stresses $\left(\sigma_{i j}\right)$ are related to displacements as

$$
\begin{aligned}
\varepsilon_{i j} & =\frac{1}{2}\left(\frac{\partial u_{i}}{\partial x_{j}}+\frac{\partial u_{j}}{\partial x_{i}}\right), \\
\sigma_{i j} & =2 \mu\left(\varepsilon_{i j}+\frac{v}{1-2 v} \varepsilon_{k k} \delta_{i j}\right),
\end{aligned}
$$


where $\mu$ is the shear modulus and $\delta_{i j}$ denotes the well-known Kronecker-delta function. Here and in the following, the customary Einstein's notation for summation over repeated subscripts is employed. The boundary tractions $t_{i}(x)$ are defined in terms of stresses as:

$$
t_{i}=\sigma_{i j} n_{j}
$$

where $n_{j}$ is the component of unit normal vector. The above equations (2.1a)-(2.4) completely describe the boundary value problems for 3D linear elasticity problems.

In the classical MFS approach, the problem considered here can be solved as follows [28,31]:

$$
\begin{aligned}
& u_{i}(x)=\sum_{n=1}^{N} \alpha_{j}^{(n)} U_{i j}\left(x, s^{(n)}\right), \\
& t_{i}(x)=\sum_{n=1}^{N} \alpha_{j}^{(n)} T_{i j}\left(x, s^{(n)}\right),
\end{aligned}
$$

where $i, j=1,2,3, x$ and $s^{(n)}$ denote the collocation point and the nth source point, respectively, $\left\{\alpha_{j}^{(n)}\right\}_{n=1}^{N}$ represent the unknown coefficients in the jth coordinate direction, and $U_{i j}(x, s)$ and $T_{i j}(x, s)$ are fundamental solutions [28] for displacements and tractions. Once all unknown coefficients $\left\{\alpha_{j}^{(n)}\right\}_{n=1}^{N}$ are solved by substituting Eqs. (2.5a) and (2.5b) into the corresponding boundary conditions (2.2a) and (2.2b), the displacements and stresses at any points inside the computational domain can then be calculated.

\section{The localized MFS for 3D elasticity problems}

In the LMFS approach, an irregular cloud of points is firstly scattered inside the whole computational domain $\Omega$. For each point $x^{(0)}$, named as the central node, the $N_{s}$ nearest nodes $\left\{x^{(m)}\right\}_{m=1}^{N_{s}}$ around $x^{(0)}$ should be found. The points $x^{(0)}$ as well as $\left\{x^{(m)}\right\}_{m=1}^{N_{s}}$ then construct a small area, which is named as the local subdomain $\Omega_{S}$ associated with the central point $x^{(0)}$ (see Fig. 1). Since $\Omega_{S}$ is a part of the original computational domain, the following FMS formulation for 3D elasticity problems should be also hold in $\Omega_{s}$ :

$$
u_{i}(x)=\sum_{n=1}^{M} \alpha_{j}^{(n)} U_{i j}\left(x, s^{(n)}\right),
$$

where $x \in \Omega_{s}, s^{(n)}$ denotes the nth source points which are uniformly distributed on a local artificial surface associated with the local subdomain $\Omega_{s}, M$ is the specified number of source points. As shown in Fig. 2, the artificial surface associated with $\Omega_{s}$ can be chosen as a sphere with radius $R_{S}$ and centered at point $x^{(0)}$. Here in our computations, $R_{S}$ is 


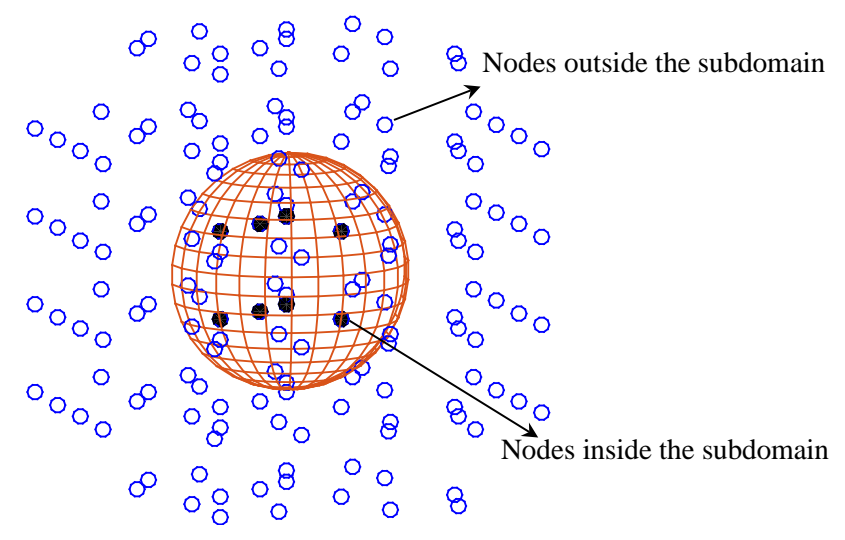

Figure 1: The schematic diagram for a local subdomain $\Omega_{s}$.

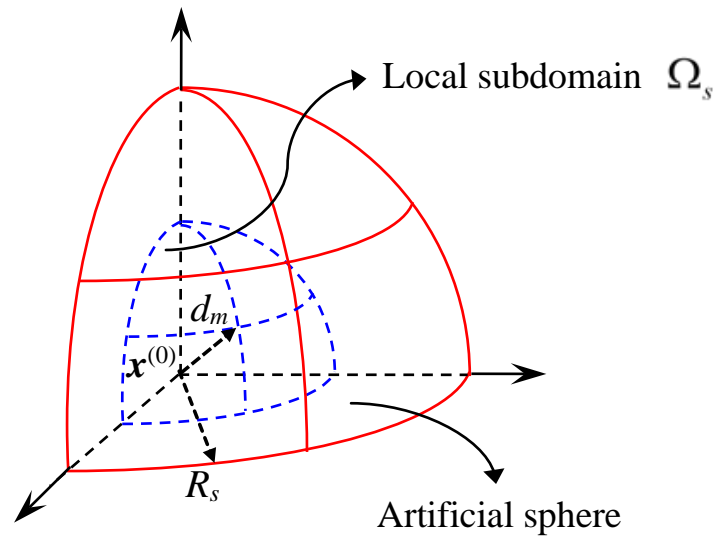

(a)

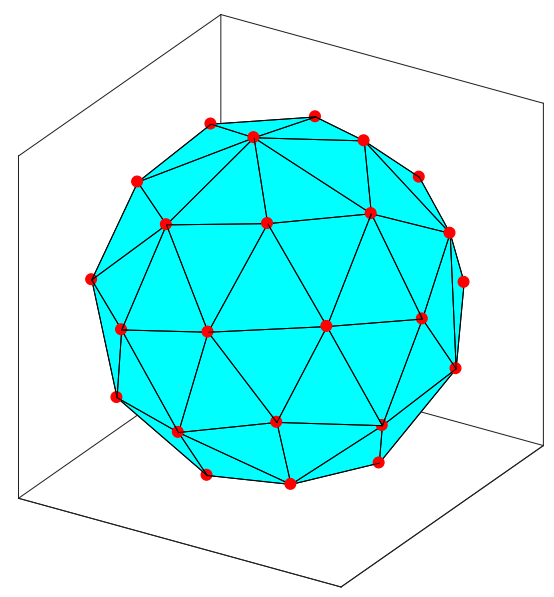

(b)

Figure 2: The schematic diagram for (a) the artificial sphere associated with $\Omega_{S}$, and (b) $M$ source points uniformly distributed along the artificial sphere surface.

taken to be $R_{s}=\lambda \times d_{m}$ where $\lambda>0$ and $d_{m}$ is the largest distance between $x^{(0)}$ and its $N_{s}$ nearest nodes.

Substituting the spatial coordinates of points $x^{(0)}$ and $\left\{x^{(m)}\right\}_{m=1}^{N_{s}}$ into Eq. (3.1) will result in the following system of equations:

$$
\left[\begin{array}{l}
u_{1} \\
u_{2} \\
u_{3}
\end{array}\right]_{3\left(N_{s}+1\right) \times 1}=\left[\begin{array}{lll}
U_{11} & U_{12} & U_{13} \\
U_{21} & U_{22} & U_{23} \\
U_{31} & U_{32} & U_{33}
\end{array}\right]_{3\left(N_{s}+1\right) \times 3 M}\left[\begin{array}{c}
\alpha_{1} \\
\alpha_{2} \\
\alpha_{3}
\end{array}\right]_{3 M \times 1},
$$


where

$$
\left[\begin{array}{lll}
u_{1} & u_{2} & u_{3}
\end{array}\right]^{T}=\left[u_{1}\left(x^{(0)}\right), \cdots, u_{1}\left(x^{\left(N_{s}\right)}\right), u_{2}\left(x^{(0)}\right), \cdots, u_{2}\left(x^{\left(N_{s}\right)}\right), u_{3}\left(x^{(0)}\right), \cdots, u_{3}\left(x^{\left(N_{s}\right)}\right)\right]^{T}
$$

are the vector of unknown displacements,

$$
\left[\begin{array}{lll}
\alpha_{1} & \alpha_{2} & \alpha_{3}
\end{array}\right]^{T}=\left[\alpha_{1}^{(n)}, \cdots, \alpha_{1}^{(M)}, \alpha_{2}^{(n)}, \cdots, \alpha_{2}^{(M)}, \alpha_{3}^{(n)}, \cdots, \alpha_{3}^{(M)}\right]^{T}
$$

is the vector of unknown coefficients, and

$$
U_{i j}=\left[\begin{array}{ccc}
U_{i j}\left(x^{(0)}, s^{(1)}\right) & \cdots & U_{i j}\left(x^{(0)}, s^{(M)}\right) \\
\vdots & \ddots & \vdots \\
U_{i j}\left(x^{\left(N_{s}\right), s^{(1)}}\right) & \cdots & U_{i j}\left(x^{\left(N_{s}\right), s(M)}\right)
\end{array}\right]_{\left(N_{s}+1\right) \times M}, \quad i, j=1,2,3,
$$

is the component of the local stiffness matrix. From Eq. (3.2), the unknown coefficients $\left[\begin{array}{lll}\alpha_{1} & \alpha_{2} & \alpha_{3}\end{array}\right]^{T}$ can be calculated as

$$
\left[\begin{array}{l}
\alpha_{1} \\
\alpha_{2} \\
\alpha_{3}
\end{array}\right]=\left[\begin{array}{lll}
U_{11} & U_{12} & U_{13} \\
U_{21} & U_{22} & U_{23} \\
U_{31} & U_{32} & U_{33}
\end{array}\right]^{-1}\left[\begin{array}{l}
u_{1} \\
u_{2} \\
u_{3}
\end{array}\right]
$$

Substituting the relation (3.4) and the coordinate of $x^{(0)}$ into Eq. (3.1) will yield the following equation:

$$
\begin{aligned}
& u_{1}^{(0)}=u_{1}\left(x^{(0)}\right)=\left[\begin{array}{lll}
h_{11} & h_{12} & h_{13}
\end{array}\right]\left[\begin{array}{l}
\alpha_{1} \\
\alpha_{2} \\
\alpha_{3}
\end{array}\right]=\left[\begin{array}{lll}
h_{11} & h_{12} & h_{13}
\end{array}\right]\left[\begin{array}{lll}
U_{11} & U_{12} & U_{13} \\
U_{21} & U_{22} & U_{23} \\
U_{31} & U_{32} & U_{33}
\end{array}\right]^{-1}\left[\begin{array}{l}
u_{1} \\
u_{2} \\
u_{3}
\end{array}\right] \\
& =\sum_{n=0}^{N_{s}}\left[\omega_{11}^{(n)} u_{1}^{(n)}+\omega_{12}^{(n)} u_{2}^{(n)}+\omega_{13}^{(n)} u_{3}^{(n)}\right] \text {, } \\
& u_{2}^{(0)}=u_{2}\left(x^{(0)}\right)=\left[\begin{array}{lll}
h_{21} & h_{22} & h_{23}
\end{array}\right]\left[\begin{array}{l}
\alpha_{1} \\
\alpha_{2} \\
\alpha_{3}
\end{array}\right]=\left[\begin{array}{lll}
h_{21} & h_{22} & h_{23}
\end{array}\right]\left[\begin{array}{lll}
U_{11} & U_{12} & U_{13} \\
U_{21} & U_{22} & U_{23} \\
U_{31} & U_{32} & U_{33}
\end{array}\right]^{-1}\left[\begin{array}{l}
u_{1} \\
u_{2} \\
u_{3}
\end{array}\right] \\
& =\sum_{n=0}^{N_{s}}\left[\omega_{21}^{(n)} u_{1}^{(n)}+\omega_{22}^{(n)} u_{2}^{(n)}+\omega_{23}^{(n)} u_{3}^{(n)}\right] \text {, } \\
& u_{3}^{(0)}=u_{3}\left(x^{(0)}\right)=\left[\begin{array}{lll}
h_{31} & h_{32} & h_{33}
\end{array}\right]\left[\begin{array}{l}
\alpha_{1} \\
\alpha_{2} \\
\alpha_{3}
\end{array}\right]=\left[\begin{array}{lll}
h_{31} & h_{32} & h_{33}
\end{array}\right]\left[\begin{array}{lll}
U_{11} & U_{12} & U_{13} \\
U_{21} & U_{22} & U_{23} \\
U_{31} & U_{32} & U_{33}
\end{array}\right]^{-1}\left[\begin{array}{l}
u_{1} \\
u_{2} \\
u_{3}
\end{array}\right] \\
& =\sum_{n=0}^{N_{s}}\left[\omega_{31}^{(n)} u_{1}^{(n)}+\omega_{32}^{(n)} u_{2}^{(n)}+\omega_{33}^{(n)} u_{3}^{(n)}\right] \text {, }
\end{aligned}
$$


where

$$
\left[h_{i 1} h_{i 2} h_{i 3}\right]=\left[U_{i 1}\left(x^{(0)}, s^{(1)}\right), \cdots, U_{i 1}\left(x^{(0)}, s^{(M)}\right), \cdots, U_{i 3}\left(x^{(0)}, s^{(1)}\right), \cdots, U_{i 3}\left(x^{(0)}, s^{(M)}\right)\right]
$$

are the vector of fundamental solutions at point $x^{(0)}$, and $\left\{\omega_{i j}^{(n)}\right\}_{i, j=1,2,3}$ denote the weighing coefficients. Eqs. (3.5a)-(3.5c) represent the relations of unknown displacements between the central node $x^{(0)}$ and its $N_{s}+1$ neighboring points. This is one of the key factors of the proposed LMFS scheme. Now we can form the final linear system of LMFS equations. Suppose a total number of $N=n_{i}+n_{b}$ points are distributed inside the entire domain, where $n_{i}$ and $n_{b}$ are numbers of interior points and boundary points, respectively. To enforce the satisfaction of Eqs. (3.5a)-(3.5c) at every interior node will yield the following linear system:

$$
\begin{aligned}
& \left(1-\omega_{11}^{(0)}\right) u_{1}^{i}-\omega_{12}^{(0)} u_{2}^{i}-\omega_{13}^{(0)} u_{3}^{i}-\sum_{n=1}^{N_{s}}\left[\omega_{11}^{(n)} u_{1}^{(n)}+\omega_{12}^{(n)} u_{2}^{(n)}+\omega_{13}^{(n)} u_{3}^{(n)}\right] \\
& =0, \quad i=1, \cdots, n_{i}, \\
& \left(1-\omega_{22}^{(0)}\right) u_{2}^{i}-\omega_{21}^{(0)} u_{1}^{i}-\omega_{23}^{(0)} u_{3}^{i}-\sum_{n=1}^{N_{s}}\left[\omega_{21}^{(n)} u_{1}^{(n)}+\omega_{22}^{(n)} u_{2}^{(n)}+\omega_{23}^{(n)} u_{3}^{(n)}\right] \\
& =0, \quad i=1, \cdots, n_{i}, \\
& \left(1-\omega_{33}^{(0)}\right) u_{3}^{i}-\omega_{31}^{(0)} u_{1}^{i}-\omega_{32}^{(0)} u_{2}^{i}-\sum_{n=1}^{N_{s}}\left[\omega_{31}^{(n)} u_{1}^{(n)}+\omega_{32}^{(n)} u_{2}^{(n)}+\omega_{33}^{(n)} u_{3}^{(n)}\right] \\
& =0, \quad i=1, \cdots, n_{i},
\end{aligned}
$$

where $\left\{u_{j}^{i}\right\}_{j=1}^{3}$ denote the displacement components of the $i$ th calculation point, $\left\{u_{j}^{(n)}\right\}_{j=1}^{3}$ stand for the displacement components at its neighboring points.

On the other hand, for boundary nodes with different boundary conditions, another linear system of equations can be obtained (displacement boundary conditions as an example):

$$
\begin{array}{ll}
u_{1}^{i}=\bar{u}_{1}^{i}, & i=n_{i}+1, \cdots, n_{i}+n_{b}, \\
u_{2}^{i}=\bar{u}_{2}^{i}, & i=n_{i}+1, \cdots, n_{i}+n_{b}, \\
u_{3}^{i}=\bar{u}_{3}^{i}, & i=n_{i}+1, \cdots, n_{i}+n_{b} .
\end{array}
$$

By combining the above equations both for interior points and boundary points, the following spare matrix system can be established:

$$
A_{3 N \times 3 N} u_{3 N \times 1}=B_{3 N \times 1},
$$

where $A$ is the coefficient matrix, $u=\left[\begin{array}{lll}u_{1} & u_{2} & u_{3}\end{array}\right]^{T}$ are the unknown displacements at every point inside the entire domain, and $B$ denotes the corresponding boundary conditions as 
well as the interior conditions (zero vector) from Eqs. (3.6a)-(3.6c). Once all displacements are calculated by solving Eq. (3.8), the stresses at any point inside the computational domain can then be calculated as:

$$
\sigma_{i j}(x)=\left[\begin{array}{lll}
s_{i j 1} & s_{i j 2} & s_{i j 3}
\end{array}\right]\left[\begin{array}{c}
\alpha_{1} \\
\alpha_{2} \\
\alpha_{3}
\end{array}\right]=\left[\begin{array}{lll}
s_{i j 1} & s_{i j 2} & s_{i j 3}
\end{array}\right]\left[\begin{array}{lll}
U_{11} & U_{12} & U_{13} \\
U_{21} & U_{22} & U_{23} \\
U_{31} & U_{32} & U_{33}
\end{array}\right]^{-1}\left[\begin{array}{l}
u_{1} \\
u_{2} \\
u_{3}
\end{array}\right]
$$

where

$$
\left[\begin{array}{lll}
s_{i j 1} & s_{i j 2} & s_{i j 3}
\end{array}\right]=\left[D_{i j 1}\left(x, s^{(1)}\right), \cdots, D_{i j 1}\left(x, s^{(M)}\right), \cdots, D_{i j 3}\left(x, s^{(M)}\right)\right]
$$

are fundamental solutions for stresses [28,32].

\section{Influence of several factors in the LMFS}

The influence of several factors on the overall accuracy of the proposed LMFS are summarized as follows:

(a) The accuracy of present method is relatively insensitive to the number of collocation nodes selected inside each of the local subdomain $\Omega_{s}$. To ensure the regularity of the matrix $U$ as shown in Eq. (3.2) [33], the number of collocation points $\left(N_{S}+1\right)$ inside $\Omega_{s}$ should be slightly larger than that of the sources $(M)$, that is $N_{s}+1 \geq M$. For simplicity, in our computations $N_{s}+1=2 M$ collocation points are chosen inside each of the local subdomain $\Omega_{s}$.

(b) The radius of the artificial sphere associated with $\Omega_{s}$. Similar to the classical MFS, the present method also requires a fictitious boundary outside $\Omega_{S}$ for the placement of the source points. Different to the classical MFS, the artificial surface here can be simply chosen as a small sphere no matter how complicated the original computational domain is, since the geometry of each of the local subdomain is relatively regular. This is one of the advantages of the present method for solving problem with very complicated geometry.

\section{$5 \quad$ Numerical results and discussions}

Three benchmark numerical examples in 3D elastostatics are presented to verify the methodologies developed in this study. The influences of several factors discussed in Section 4 on the overall accuracy of the present method are carefully studied. For the ease of comparison, the analytical solutions for displacements and stresses are taken to 
be:

$$
\begin{array}{llrl}
u_{1}(x) & =\frac{1-2 v}{2 \mu \nu} x_{1}, & u_{2}(x)=\frac{1-2 v}{2 \mu v} x_{2}, & u_{3}(x)=-\frac{1-2 v}{2 \mu v} x_{3}, \\
\sigma_{11}(x)=\frac{1-v}{v}, & \sigma_{22}(x)=\frac{1-v}{v}, & \sigma_{33}(x)=-\frac{1-3 v}{v} \\
\sigma_{12}(x)=\sigma_{13}(x)=\sigma_{23}(x)=0, & &
\end{array}
$$

where $v=0.2$ and $\mu=1.3889 \times 10^{5} \mathrm{MPa}$. All the numerical problems studied here are subjected to mixed-type boundary conditions. To study the accuracy of the proposed method, the following $L_{2}$ error (relative/global error) is adopted:

$$
E_{\text {global }}=\left[\sum_{k=1}^{N_{\text {total }}}\left[I_{\text {numer }}(k)-I_{\text {exact }}(k)\right]^{2}\right]^{1 / 2} /\left[\sum_{k=1}^{N_{\text {total }}}\left[I_{\text {exact }}(k)\right]^{2}\right]^{1 / 2},
$$

where $I_{\text {numer }}$ and $I_{\text {exact }}$ stand for the numerical and analytical solutions, respectively, $N_{\text {total }}$ is the total number of points tested.

\subsection{Test problem 1: stress analysis in a cubic domain}

As shown in Fig. 3, stress analysis in a cubic domain $\Omega=(0,1)^{3} \subset R^{3}$ is considered. The tractions are specified at the surface $\left(x_{1}=1, x_{2}, x_{3}\right)$ while the displacements are prescribed on the remaining surfaces. A total number of $N=2057$ uniformly distributed points are selected inside the entire domain. The parameters $R_{s}$ and $M$ associated with each of the local subdomain $\Omega_{s}$ are chosen as $R_{s}=8 \times d_{m}$ and $M=30$. Fig. 4 displays the relative errors of the calculated stresses $\sigma_{11}(x)$ and $\sigma_{33}(x)$ at points distributed in a square domain $\left\{\left(x_{1}, x_{2}, 0.5\right) \mid 0 \leq x_{1}, x_{2} \leq 1\right\}$. As shown in Fig. 4, numerical results calculated by using the proposed method are in excellent agreement with their corresponding analytical solutions, with the largest relative less than $6 \times 10^{-5}$.

Table 1 illustrates the sensitivity of the numerical results with respect to the number of source points $(M)$ associated with $\Omega_{s}$, with $R_{s}=8 \times d_{m}$. As can be seen from Table 1 , the numerical results for both $\sigma_{11}, \sigma_{22}, \sigma_{33}, u_{1}$ and $u_{2}$ are rapidly convergent as the number of source points increases. Next, in Table 2, we fix $M=30$ and study the effect of the size of the artificial sphere $R_{S}$ on the accuracy of the present method. It can be seen from Table 2 that the accuracy of the present method improves steadily as the radius of the fictitious boundary increases. In Tables 1 and 2, the global errors are calculated by using Eq. (5.2).

Fig. 5 illustrates the efficiency of the present method for large-scale simulations. For comparison, numerical results calculated by using the classical MFS, BEM (with constant elements) and the generalized finite difference method [34,35] are also provided. As shown in Fig. 5, due to the memory limitations, the classical MFS and BEM are valid only when the total number of points is less than 8,000 . In contrast, the present method is valid for the largest model with up to 100,000 points. This illustrates significant advantage of the proposed method in the savings of CPU-times compared with the MFS and the BEM. 


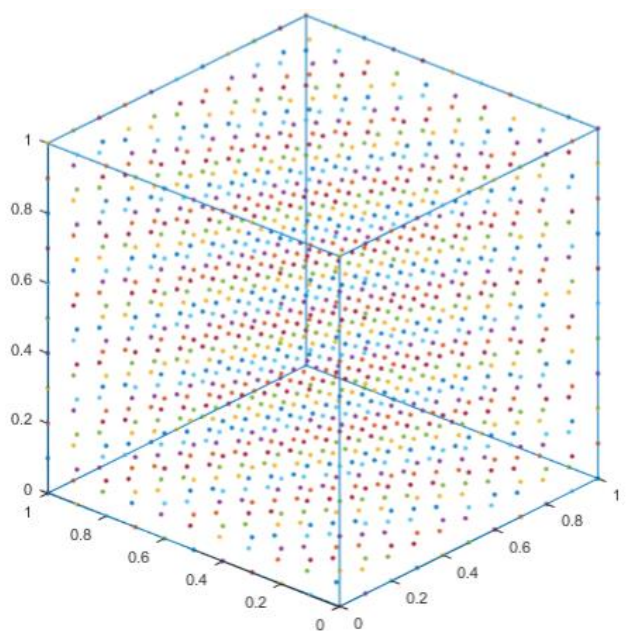

Figure 3: Geometry of the problem and the nodes distribution of the LMFS model.

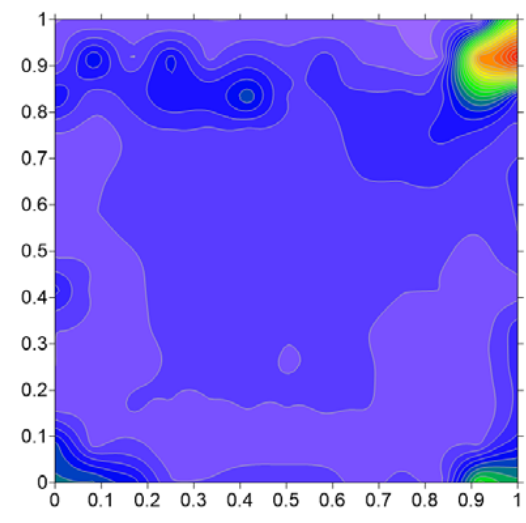

(a) Stresses $\sigma_{11}(x)$

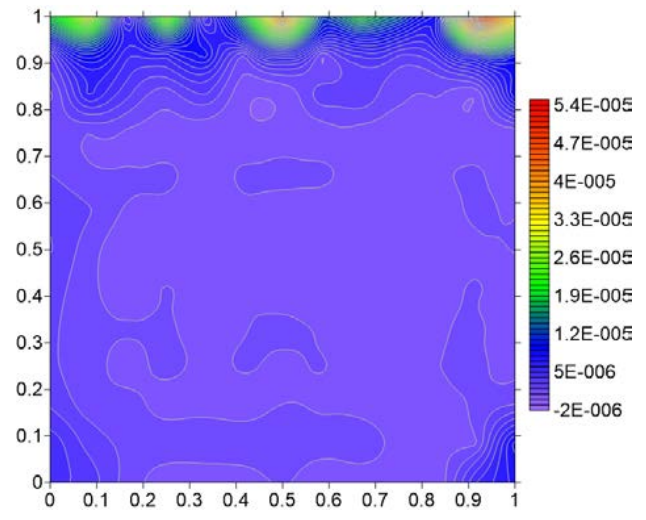

(b) Stresses $\sigma_{33}(x)$

Figure 4: Relative errors of the calculated stress at points located in the surface $x_{3}=0.5$.

Table 1: Relative errors for stresses as functions of different number of sources $M$ in each subdomain $\Omega_{S}$.

\begin{tabular}{|c|c|c|c|c|c|c|}
\hline \multicolumn{2}{|c|}{ Number of sources $(M)$} & $M=20$ & $M=30$ & $M=40$ & $M=50$ & $M=60$ \\
\hline$\sigma_{11}$ & Global errors & $8.889 \times 10^{-3}$ & $1.176 \times 10^{-4}$ & $3.374 \times 10^{-5}$ & $2.556 \times 10^{-5}$ & $9.953 \times 10^{-6}$ \\
\hline$\sigma_{22}$ & Global errors & $5.331 \times 10^{-3}$ & $9.327 \times 10^{-5}$ & $1.923 \times 10^{-5}$ & $1.593 \times 10^{-5}$ & $8.754 \times 10^{-6}$ \\
\hline$\sigma_{33}$ & Global errors & $1.791 \times 10^{-2}$ & $2.499 \times 10^{-4}$ & $1.112 \times 10^{-4}$ & $5.402 \times 10^{-5}$ & $2.125 \times 10^{-5}$ \\
\hline$u_{1}$ & Global errors & $6.845 \times 10^{-3}$ & $1.658 \times 10^{-4}$ & $7.673 \times 10^{-5}$ & $4.879 \times 10^{-5}$ & $1.896 \times 10^{-5}$ \\
\hline$u_{2}$ & Global errors & $8.943 \times 10^{-3}$ & $8.783 \times 10^{-5}$ & $2.892 \times 10^{-5}$ & $1.984 \times 10^{-5}$ & $1.087 \times 10^{-5}$ \\
\hline & times (second) & 7.738 & 18.296 & 25.153 & 32.819 & 47.742 \\
\hline
\end{tabular}




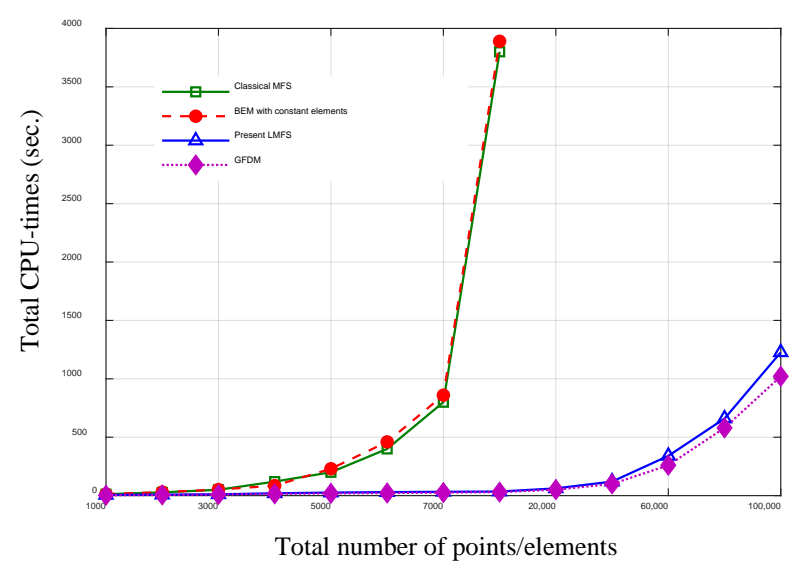

Figure 5: Comparison of the CPU-times for the classical MFS, BEM, GFDM and the LMFS models.

Table 2: Relative errors for stresses as functions of source radius Rs in each subdomain $\Omega_{S}$.

\begin{tabular}{||cc|ccccc||}
\hline \multicolumn{2}{|c|}{ Source radius $\left(R_{S}\right)$} & $R_{S}=5 \times d_{m}$ & $R_{s}=8 \times d_{m}$ & $R_{s}=12 \times d_{m}$ & $R_{s}=15 \times d_{m}$ & $R_{s}=18 \times d_{m}$ \\
\hline$\sigma_{11}$ & Global errors & $1.650 \times 10^{-3}$ & $1.176 \times 10^{-4}$ & $3.663 \times 10^{-5}$ & $1.378 \times 10^{-5}$ & $5.492 \times 10^{-6}$ \\
$\sigma_{22}$ & Global errors & $9.882 \times 10^{-4}$ & $9.327 \times 10^{-5}$ & $2.891 \times 1010^{-5}$ & $1.095 \times 10^{-5}$ & $4.045 \times 10^{-6}$ \\
$\sigma_{33}$ & Global errors & $2.869 \times 10^{-3}$ & $2.499 \times 10^{-4}$ & $7.593 \times 1010^{-5}$ & $2.863 \times 10^{-5}$ & $1.085 \times 10^{-6}$ \\
$u_{1}$ & Global errors & $5.895 \times 10^{-3}$ & $1.658 \times 10^{-4}$ & $4.982 \times 10^{-5}$ & $2.985 \times 10^{-5}$ & $1.549 \times 10^{-5}$ \\
$u_{2}$ & Global errors & $3.784 \times 10^{-3}$ & $8.783 \times 1010^{-4}$ & $6.783 \times 10^{-5}$ & $3.115 \times 10^{-5}$ & $1.003 \times 10^{-5}$ \\
\hline
\end{tabular}

\subsection{Test problem 2: stress analysis in a mechanical component}

Next, we consider the stress analysis in a classical mechanical component, as shown in Fig. 6. The principal dimension of the problem is $2 \mathrm{~m}$ in length, $1.2 \mathrm{~m}$ in width, and $1 \mathrm{~m}$ in height. A total number of 19,525 irregularly distributed nodes are selected inside the entire domain. The problem is subjected to mixed-type boundary conditions, where the tractions are given on the right-half surface of the domain $\{-1 m \leq x \leq 0 m\}$, while the displacements are imposed on the remaining surface.

Figs. 7(a) and (b) illustrate the effect of the number of source points $(M)$ and the radius of the fictitious sphere $\left(R_{S}\right)$ on the overall accuracy of the present method. In Fig. 7(a), we fix $R_{s}=4$, and show how the global error decays as $M$ increases. We can see that the LMFS results are stable, accurate and rapidly convergent as the number of source points increases. Fig. 7(b) investigates the error variations with respect to $R_{s}$. Similarly, the proposed LMFS results are rapidly convergent as $R_{S}$ increases.

\subsection{Test problem 3: stress analysis in a drone-shaped solid}

Finally, we consider the stress analysis through a drone-shaped solid, as shown in Fig. 8. The problem considered here has an overall dimension $3 \mathrm{~m} \times 0.9 \mathrm{~m} \times 3.5 \mathrm{~m}$. This $3 \mathrm{D}$ model 


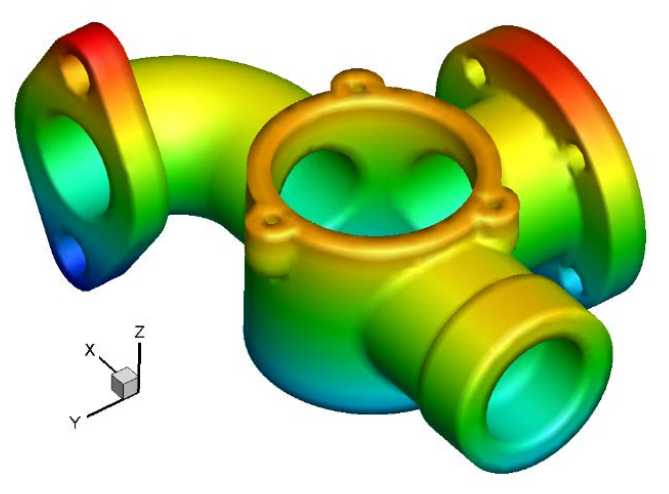

(a) Geometry of the problem

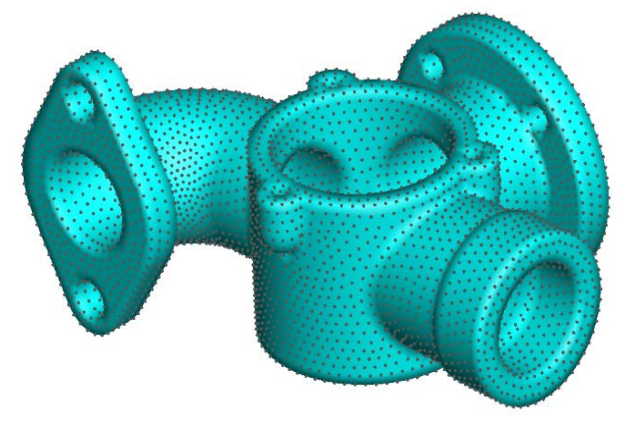

(b) Nodes distribution of the LMFS model

Figure 6: Geometry of the problem (a) and the nodes distribution of the LMFS model (b).

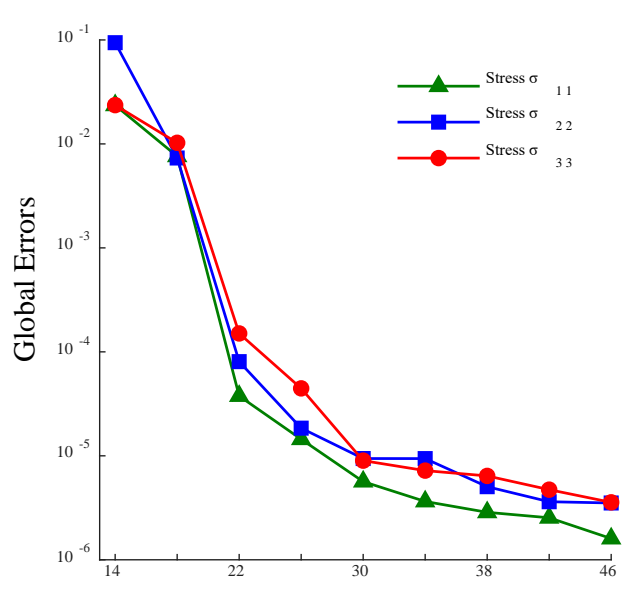

(a) Number of source points $M$

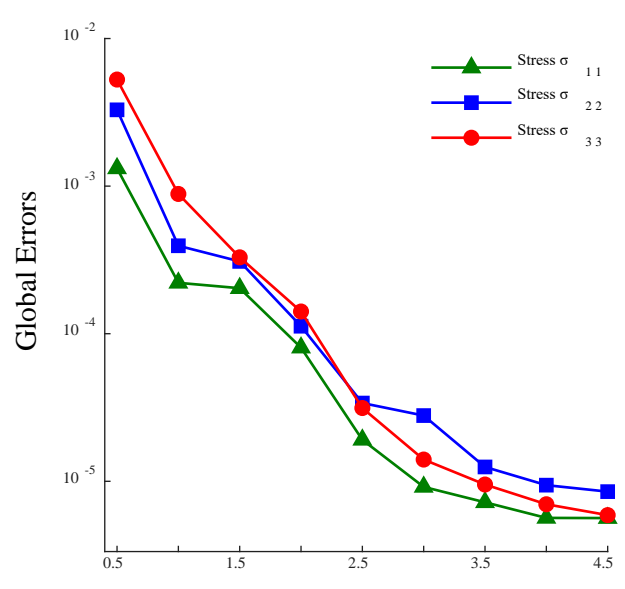

(b) The radius of the fictitious sphere

Figure 7: Relative error curves for stresses, as functions of: (a) different number of source points $(M)$ with $R_{s}=4$; and (b) various values for the source radius $R_{s}$ with $M=30$.

is subjected to mixed-type boundary conditions, where the tractions are prescribed on the surface $\{-1.5 m \leq x \leq 0 m\}$, while the displacements are imposed on the remaining surface. To investigate the convergence of the proposed method, the code of the proposed LMFS is run four times which are respectively: (a) $N=5,762, M=20$; (b) $N=19,525$, $M=25$; (c) $N=35,759, M=30$; (d) $N=89,247, M=35$, where $N$ is the number of points selected inside the whole domian. Fig. 9 shows the contours of stress results $\left(\sigma_{11}\right)$ at points along the surface of the domain. The radius $\left(R_{s}\right)$ of the artificial sphere is fixed as $R_{s}=4$. It can be seen from this figure that the relative errors of the calculated stresses are decreased rapidly with increasing number of $N$ and $M$. Although not presented, it 


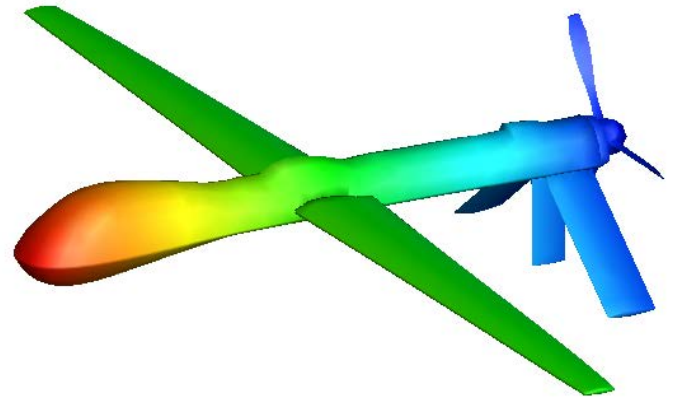

(a) Geometry of the problem

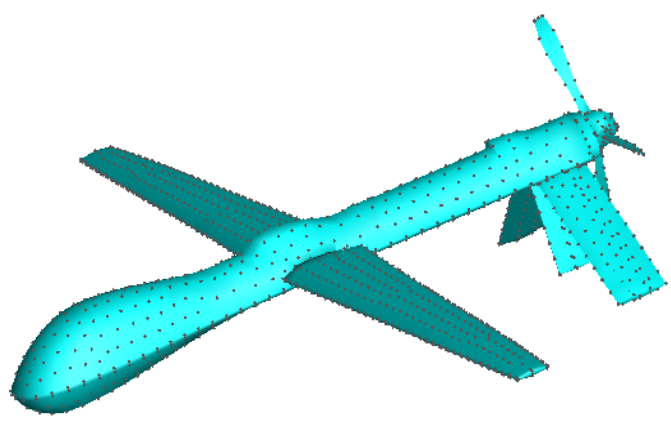

(b) Nodes distribution of the LMFS model

Figure 8: Geometry of the problem (a) and the nodes distribution of the LMFS model (b).

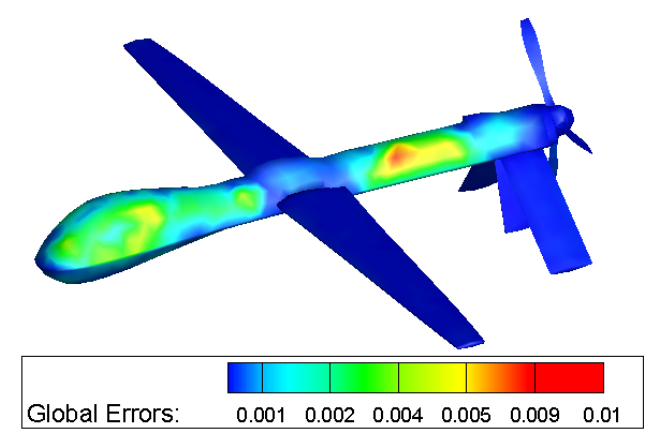

(a) $N=5,762, M=20$

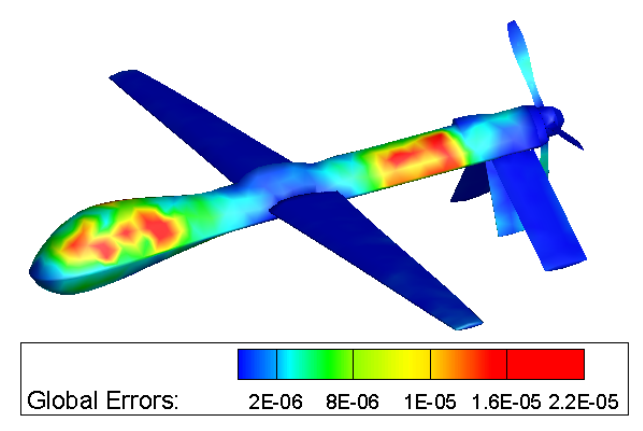

(c) $N=35,759, M=30$

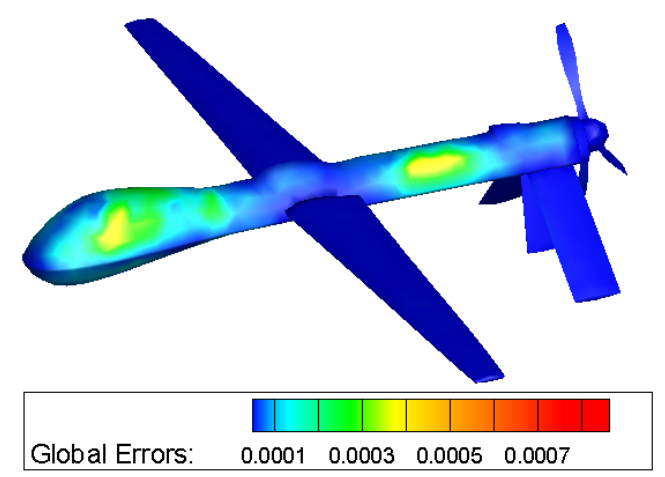

(b) $N=19,525, M=25$

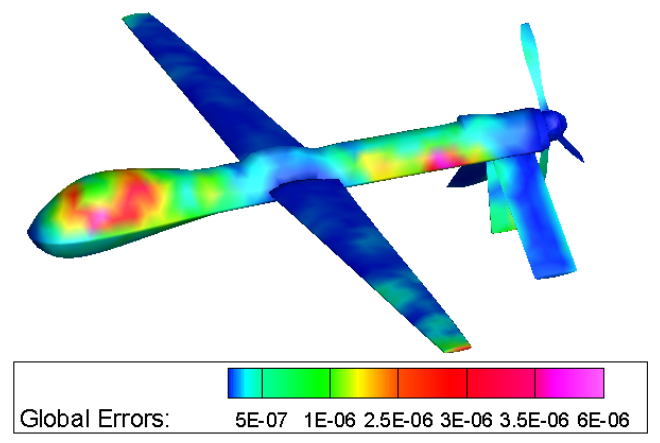

(d) $N=89,247, M=35$

Figure 9: Relative errors of the computed stresses $\sigma_{11}$ retrieved on the surface of the computational domain.

is reported that numerous other numerical experiments have been performed and the similar conclusions have been drawn. 


\section{Concluding remarks}

In this paper, we investigate the use of the localized method of fundamental solutions (LMFS) for the large-scale simulations of 3D elasticity problems. The coefficient matrix of the present method is sparse, which makes the method possible to perform largescale simulations on a desktop computer. Though the method has been developed in the context of 3D elasticity problems, extension of the method to many other problems in computational mechanics is fairly straightforward. It is hoped that the insights presented in this paper will help to spur more interest in and launch more investigations into the use of the LMFS for the numerical simulations.

\section{Acknowledgements}

The work described in this paper was supported by the National Natural Science Foundation of China (Nos. 11872220, 11772119), and the Natural Science Foundation of Shandong Province of China (Nos. ZR2017JL004, 2019KJI009). The first author would like to thank the Alexander von Humboldt Foundation for the Humboldt Research Fellowship at the Chair of Structural Mechanics, University of Siegen, Germany.

\section{References}

[1] A. KARAgEORghis, D. LeSNiC, AND L. MARIN, The method of fundamental solutions for an inverse boundary value problem in static thermo-elasticity, Comput. Struct., 135 (2014), pp. 32-39.

[2] C. S. Chen, M. A. GolberG, AND Y. C. Hon, The method of fundamental solutions and quasi-Monte-Carlo method for diffusion equations, Int. J. Numer. Methods Eng., 43 (1998), pp. 1421-1435.

[3] A. ZHANG, Y. GU, Q. HuA, W. CHEN, AND C. ZHANG, A regularized singular boundary method for inverse Cauchy problem in three-dimensional elastostatics, Adv. Appl. Math. Mech., 10 (2018), pp. 1459-1477.

[4] C. J. S. Alves, AND C. S. CHEN, A new method of fundamental solutions applied to nonhomogeneous elliptic problems, Adv. Comput. Math., 23 (2005), pp. 125-142.

[5] Q. G. LIU, AND B. S̆ARLER, Non-singular method of fundamental solutions for elasticity problems in three-dimensions, Eng. Anal. Bound. Elem., 96 (2018), pp. 23-35.

[6] L. MARIN, AND C. CIPU, Non-iterative regularized MFS solution of inverse boundary value problems in linear elasticity: A numerical study, Appl. Math. Comput., 293 (2017), pp. 265-286.

[7] Z.-J. Fu, Q. XI, W. CHEN, AND A. H. D. CHENG, A boundary-type meshless solver for transient heat conduction analysis of slender functionally graded materials with exponential variations, Comput. Math. Appl., 76 (2018), pp. 760-773.

[8] B. SARLER, AND Q. G. LIU, Non-singular method of fundamental solutions for two-dimensional isotropic elasticity problems, Comput. Model. Eng. Sci., 91 (2013), pp. 235-266.

[9] Y. GU, X. HE, W. CHEN, AND C. ZHANG, Analysis of three-dimensional anisotropic heat conduction problems on thin domains using an advanced boundary element method, Comput. Math. Appl., 75 (2018), pp. 33-44. 
[10] J. R. BERGER, AND A. KARAGEORGHIS, The method of fundamental solutions for layered elastic materials, Eng. Anal. Bound. Elem., 25 (2001), pp. 877-886.

[11] B. SARLER, Solution of potential flow problems by the modified method of fundamental solutions: Formulations with the single layer and the double layer fundamental solutions, Eng. Anal. Bound. Elem., 33 (2009), pp. 1374-1382.

[12] J. LI, AND W. CHEN, A modified singular boundary method for three-dimensional high frequency acoustic wave problems, Appl. Math. Model., 54 (2018), pp. 189-201.

[13] F. WANG, C.-S. LIU, AND W. QU, Optimal sources in the MFS by minimizing a new merit function: Energy gap functional, Appl. Math. Lett., 86 (2018), pp. 229-235.

[14] C. S. CHEN, A. KARAGEORGHIS, AND Y. LI, On choosing the location of the sources in the MFS, Numer. Algoritms, 72 (2016), pp. 107-130.

[15] Y. J. LIU, Y. X. LI, AND S. HUANG, A fast multipole boundary element method for solving twodimensional thermoelasticity problems, Comput. Mech., 54 (2014), pp. 821-831.

[16] L. MARIN, An invariant method of fundamental solutions for two-dimensional steady-state anisotropic heat conduction problems, Int. J. Heat. Mass Transfer, 94 (2016), pp. 449-464.

[17] L. MARIN, A. KARAGEORGHIS, AND D. LESNIC, Regularized MFS solution of inverse boundary value problems in three-dimensional steady-state linear thermoelasticity, Int. J. Solids Struct., 91 (2016), pp. 127-142.

[18] W. QU, Y. ZHANG, AND C.-S. LIU, A new regularized boundary integral equation for threedimensional potential gradient field, Adv. Eng. Software, 96 (2016), pp. 83-90.

[19] Y. J. LIU, N. NishimURA, AND Z. H. YAO, A fast multipole accelerated method of fundamental solutions for potential problems, Eng. Anal. Bound. Elem., 29 (2005), pp 1016-1024.

[20] Y. GU, H. GAO, W. CHEN, C. LIU, C. ZHANG, AND X. HE, Fast-multipole accelerated singular boundary method for large-scale three-dimensional potential problems, Int. J. Heat Mass Transfer, 90 (2015), pp. 291-301.

[21] J. LIN, C. S. CHEN, C.-S. LIU, AND J. LU, Fast simulation of multi-dimensional wave problems by the sparse scheme of the method of fundamental solutions, Comput. Math. Appl., 72 (2016), pp. 555-567.

[22] J. LiN, S. Y. ReUtSKIY, AND J. LU, A novel meshless method for fully nonlinear advection-diffusion-reaction problems to model transfer in anisotropic media, Appl. Math. Comput., 339 (2018), pp. 459-476.

[23] W. QU, A high accuracy method for long-time evolution of acoustic wave equation, Appl. Math. Lett., 98 (2019), pp. 135-141.

[24] Z.-J. FU, J. ZHANG, P.-W. LI, AND J.-H. ZHENG, A semi-Lagrangian meshless framework for numerical solutions of two-dimensional sloshing phenomenon, Eng. Anal. Boundary Elements, 112 (2020), pp. 58-67.

[25] C. M. FAN, Y. K. HuAnG, C. S. Chen, AND S. R. KuO, Localized method of fundamental solutions for solving two-dimensional Laplace and biharmonic equations, Eng. Anal. Boundary Elements, 101 (2019), pp. 188-197.

[26] Y. GU, C.-M. FAN, W. QU, AND F. WANG, Localized method of fundamental solutions for largescale modelling of three-dimensional anisotropic heat conduction problems-Theory and MATLAB code, Comput. Struct., 220 (2019), pp. 144-155.

[27] Y. GU, C.-M. FAN, AND R.-P. XU, Localized method of fundamental solutions for large-scale modeling of two-dimensional elasticity problems, Appl. Math. Lett., 93 (2019), pp. 8-14.

[28] Y. GU, W. CHEN, H. GAO, AND C. ZHANG, A meshless singular boundary method for threedimensional elasticity problems, Int. J. Numer. Methods Eng., 107 (2016), pp. 109-126.

[29] G. R. LIU, A point assembly method for stress analysis for two-dimensional solids, Int. J. Solids 
Struct., 39 (2002), pp. 261-276.

[30] W. HU, Y. GU, C. ZHANG, AND X. HE, The generalized finite difference method for an inverse boundary value problem in three-dimensional thermo-elasticity, Adv. Eng. Software, 131 (2019), pp. 1-11.

[31] L. MARIN, A meshless method for solving the cauchy problem in three-dimensional elastostatics, Comput. Math. Appl., 50 (2005), pp. 73-92.

[32] Y. GU, C. ZHANG, W. QU, AND J. DING, Investigation on near-boundary solutions for threedimensional elasticity problems by an advanced BEM, Int. J. Mech. Sci., 142-143 (2018), pp. 269275.

[33] Y. GU, W. QU, W. CHEN, L. SONG, AND C. ZHANG, The generalized finite difference method for long-time dynamic modeling of three-dimensional coupled thermoelasticity problems, J. Comput. Phys., 384 (2019), pp. 42-59.

[34] Y. GU, AND H. SUN, A meshless method for solving three-dimensional time fractional diffusion equation with variable-order derivatives, Appl. Math. Model., 78 (2020), pp. 539-549.

[35] Y. WANG, Y. GU, AND J. LIU, A domain-decomposition generalized finite difference method for stress analysis in three-dimensional composite materials, Appl. Math. Lett., 104 (2020), pp. 106226. 